\title{
Algorithm of Remote Monitoring ECG Using Mobile Phone: Conception and Implementation.
}

\author{
Rachid, Merzougui; Mohammed, Feham \\ Faculty of Engineering Science of Tlemcen, Algeria \\ STIC Laboratory \\ Chetouane Tlemcen Algeria \\ j2me_com@hotmail.com,m_feham@mail.univ-tlemcen.dz \\ tel. +213551 629402, fax. +213043285685
}

\begin{abstract}
Researches in the medical remote monitoring at home have taken a great consideration and care in wireless communication these last years.

The set of these researches is linked to the aging population and lack of infrastructures of reception for persons exposed to accident's risks in their daily life or degradation of their health in short time. The principal objective is to permit a taking off for medical and social dependant people as aged ones, handicapped, in order to the adaptation with their environment domestically and make up their incapacities. In this case, it is indispensable to make a diagnostic in a real time and managed really the given data of patients between medical actors with the permanent security insurance of highly risky patients.

Furthermore, the need to make a speed diagnostic of patients and to detect their health state with efficacy permits the gain of time in their taking off.

The stakes of setting up such systems are numerous, so much for patients, medical staff and the society generally. So, it is useful to detect and prevent the occurrence of critical situations of a person at home, implying the transmission of message and alarms to actors concerned and ready to intervene in case of necessity.

Our attention has been focused on the choice of a relevant work. It concerns an application on a mobile terminal (MIDlet) for monitoring patient in a non-hospital setting. This paper recalls a complete architecture of an economic wireless transmission system with the implementation of an effective algorithm, adapted to the mobile terminal, allowing to the doctor to have the results of analysis of ECG data wirelessly.
\end{abstract}

Keywords: Remote Monitoring, Mobile, J2ME, ECG.

\section{Introduction}

Telemedicine appears to be a medical reality: it has already imposed the use of portable units as mobile phones. The recent technological advances of mobile telecommunications networks applied to the medical domain (medical imaging, debit of transmission, confidentiality of data, the conviviality of systems, etc.) and the miniaturization of devices open perspectives for medical development of remote monitoring in term of acroissement of the efficiency and the care's quality, the knowledge's sharing or reduction of public health cost. These new technologies have led to the emergence of a wide variety of new ways for users to access and use information which interests it anywhere and anytime [1]. Then, today a simple mobile phone can contribute effectively to safeguard of the human lives. This paper describes an application using mobile wireless networks to treat and monitor the state of patient and aged ones at home.

The suggested solution is an implement of an algorithm which transmits the data of the patient via a wireless communication in the purpose to exploit a mobile phone for medical monitoring (detection, calculation of cardiac frequency, visualization of ECG signal on the screen of the mobile phone...).

The majority of the works undertaken in this field carry out the analysis of the signals on large server (great capacities, better resolutions...).

We suggest in this article to introduce the complete analysis of ECG signal for the cardiac persons on a simple mobile phone by respecting its necessary constraints.

Thus the orientation of our works in this sense was dictated by mobile networks services, simplicity of 
management and adaptation to the context of mobility with a low cost of exploitation.

In following sections, we present different subjects concerning the solution of detecting critical situations on limited resources by an adaptation strategy to design and develop health services.

Indeed, it is necessary at first to formulate precisely the problem to identify the areas of research which effectively require to be addressed.

\section{Problem}

In the context of this paper, we are particularly focused on the carried out operations in the processing and analysis of the received medical signals from the installed sensors at home. This step is fundamental to an effective exploitation of the potentialities for collecting a big amount of data which improves monitoring to ensure a permanent safety of patients at home and prevents a degradation of their Health state. The extracted information on the patient's situation must be relevant for help to diagnosis. This approach is significant in comparison with the great quantity and the diversity of the data, as well as the need of a personalized treatment for each patient.

The constraints of this work concern in particular to the need for an approach focused on the characterization and determination of the parameters of specific ECG signal of each person. The habits of daily life as well as physiological characteristics vary according to individuals. The complexity of the problem resides in a great interindividual variability of the recorded data, and also in broad intra-individual possible modifications being given the often not very foreseeable aspect of human behaviors [2], [3].

The problem also is posed on the level implementation of an efficient algorithm intended to solve all the preceding constraints and adapted to mobile phones. This implementation requires many constraints (low resource calculation, memory capacity, resolution...) to run properly.

In this context, the considered study leads to an inexpensive solution, efficient and comfortable for patients at any time and anywhere, provided that they have a mobile terminal. Indeed, they could benefit of a medical monitoring security, without the inconvenience and without excessive expenditures.

\section{Our vision}

The cost of the health represents a considerable weight in the economic balance sheet on international scale. Also, in many countries, the aging or psychological shocks of the population tends to increase the number of people strongly requiring medical monitoring even more or less intensive care and of this fact the global cost of medical care.

As all the technologies, the mobile telephony is evolved, and actually the offered possibilities are more important than ten years ago. But indeed as often, the majority of users use only the basic functions, phoning and sending messages (SMS), what have already allowed envisaging multitude applications.

In our research task on the subject, we propose the exploitation of the mobile phone in other applications apart from the vocal communications.

The idea is to divert these devices of their basic function to make them useful for medical supervision.

Whereas ten years before, such a taking off would have required large means as well as a large infrastructure, today a simple mobile phone can effectively contribute to the protection of human lives.

\subsection{Platform system and functions}

The considered system (Figure 1) allows a patient to be in contact, at any time, with his doctor for medical monitoring.

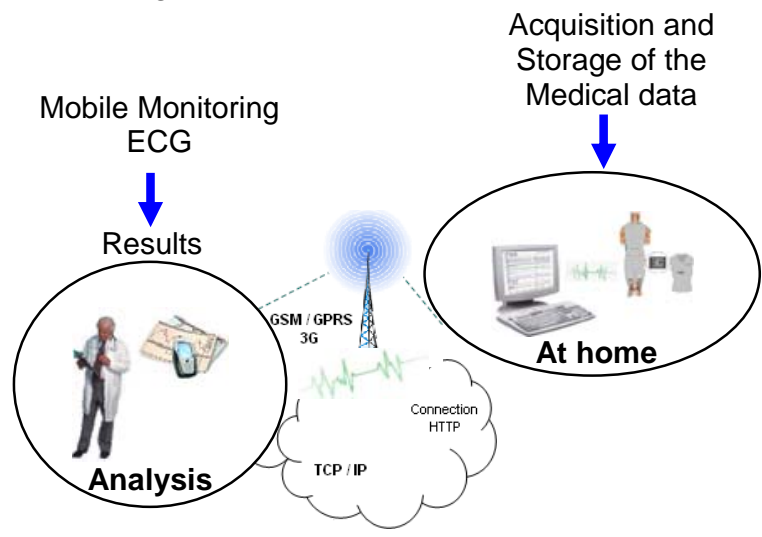

Figure1: Architecture of the platform system.

The implemented application on a mobile phone, functions on all mobile terminals or PDAs equipped with a J2ME virtual machine. This algorithm allows communications GSM / GPRS with other devices. In this case, the transmitted data concerns the ECG signal collected on remote sites. The procedure 
consists to implement on a mobile device the following operations:

- Display of 5000 samples (amplitudes: mV) of signal ECG of the patient.

- Detection of the peaks $\mathrm{R}$ (times between each two peaks, cardiac frequency).

- Calculation of QRS durations.

- Visualization of ECG signal with 5000 samples on the screen of mobile terminal.

- Possibility to zoom the ECG signal.

The first part which must be realized concerns the collection of remotely medical data on the mobile terminal. These data are generated by sensors installed at patient's home.

Then it is necessary to make connection between the medical supervision device (mobile phone) and the remote monitoring system at patient's home, so that they can exchange their data.

An adaptation strategy to medical context was followed to generate analysis results of calculation algorithm implemented on doctor's mobile terminal (detail in the following sections). Thus, the doctor is able to check the result of the diagnosis obtained by this algorithm.

\subsection{Choice of technology}

The analysis carried out made it possible to better understand the principal protocols which intervene in the development of our application.

After studying the various technologies, in terms of remote exploitation of the stored data on a computer at home, the most adapted solution consists to use a simple mobile phone linked by a GSM / GPRS to this database. This technology is easy and rapid to be implemented.

This implies the existence of an http connection between doctor's mobile terminal and monitoring home automation system via a WAP gateway to recover data of ECG signal. This choice is dictated by the following characteristics: [4]

- Http is obligatorily implemented on all terminals MIDP (J2ME).

- Http is independent of the network.

- The port 80 of the http protocol is more easily working on the firewall.

- Http protocol is implemented by default in J2ME package. Other protocols are not necessarily available.

The transfer of the medical data of a patient at home on the mobile phone is based on a communication
WSP/ Http. As its name suggests Wireless Session Protocol (WSP), session layer allows the connection setting to make transactions. Thus it allows the layer application to profit from two different types of sessions:

- Connected session mod which the layer session will interact with the layer transaction.

- No-Connected session mod where the session layer will act directly at the transport layer for sending brutes' datagram.

WSP as a whole is equivalent to the http protocol. We find moreover many identical implementations to the http in WSP.

\subsection{Choice of development environments}

The recording and the acquisition of the ECG data were carried out using MATLAB.

Java applications have been implemented under NetBeans IDE environment.

A simulation tool Sun Java тм Wireless Toolkit 2.5 for CLDC was exploited to examine all the possible wireless communications. It allows applications on devices with low calculation resources such as a mobile phone [5].

The choice of Java is justified by the different problems associated to coding in $\mathrm{C}++$ on Symbian operating system:

- Management of the memory: for the majority of applications, Java system seems to be sufficient.

- Environment of execution: the proposed options on executable Java as protections for downloading or secure execution are free, whereas $\mathrm{C}++$ it is necessary to develop them, test them and maintain them.

- Perpetuity: Java seems to have been accepted for the development of applications on mobile phones. The future developments will make Java perhaps as fast as $\mathrm{C}++$.

Thus for that principal reason, Java was chosen in our project, but it is necessary to mention that both environments can be used [6].

\section{Implementation of proposed model}

As mentioned before, our implementation achieves the medical service which provides the continuity of remote monitoring at home and immediate alarms to deal with the patient in the event of need.

The schedule of conditions of our project consists of: 
- The implementation of this service requires the development of two distinct applications:

- A first to be installed on the mobile phone to detect and treat the critical situations of patient via wireless support.

- A second function on the PC at home in order to acquire and record data relating to the patient.

- To program the application in a language which is most portable possible, the algorithm must be simple to use and install.

- To program a user interface of high quality.

The suggested model is also based on techniques of programming adapted not only to the limited resources (devices of medical supervision) but also to the generated heterogeneous parameters. What allows in particular showing the diversity of profiles of persons and types of generated situations, including the simulation of "normal" modifications and disturbing of behavior.

The following paragraphs present (1) Development on mobile phone, (2) Development on PC, (3) the simulation of the proposed model.

\subsection{Development on mobile phone}

J2ME is a collection of technologies and specifications which are conceived for various parts of the market of the small devices. The principal part of platform J2ME is composed of two different configurations (Figure 2): Connected Device Configuration (CDC) and Connected Limited Device Configuration (CLDC) [4].

A configuration defines the central libraries of Java technology and virtual storage capacities of the device. CLDC is adapted to recent mobile phones. This configuration is useful for our application. To still note, that in the case of J2ME, the virtual machine is called KVM for Kilobyte Virtual Machine.

At the top of the configurations (Figure 2), there are the profiles which define the functionalities in each specific category of devices. The "Mobile Information Device Profile" (MIDP) is a profile for the mobile devices using configuration CLDC, like the mobile phones. Profile MIDP specifies the functionalities like the use of the interface user, the persistence of storage, the setting in network and the model of application.

On the majority of the current phones, J2ME is composed of configuration CLDC and profile MIDP.

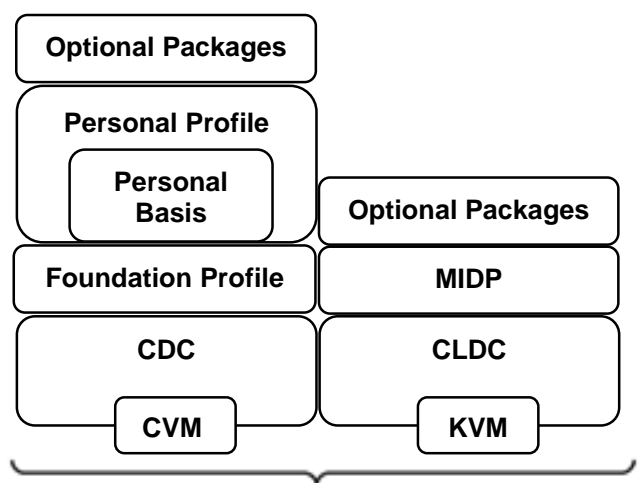

Figure 2: Architecture of J2ME.

In addition to standard MIDP (Figure 2), additional (optional) packages can be added according to the devices, allowing the use of their specificities.

As these options are typically reserved to mobile phones, it was natural to not integrate them directly in the profile MIDP.

So, the development of our application on mobile phones is based on the use of configuration CLDC and profile MIDP. In addition to these two standard elements, we have exploited some optional packages such as WMA for the management of services SMS / MMS and Web Services API. The libraries necessary for the implementation for each component of J2ME are as follows: [6]

API MIDP: is, till now, what is installed on mobiles "compatible J2ME":

-javax.microedition.Icdui : it provides the graphic components necessary to the creation of applications. -javax.microedition.midlet: It provides the component application as well as the primitives managing the life of the application.

-javax.microedition.rms: A possibility of storage of information on the terminal.

\section{API CLDC:}

Javax.microedition.io: It contains the classes making it possible to connect itself via TCP/IP or $U D P$. The principal object of this package is the class Connector [4].

This network part determines which means used to communicate medical data. 


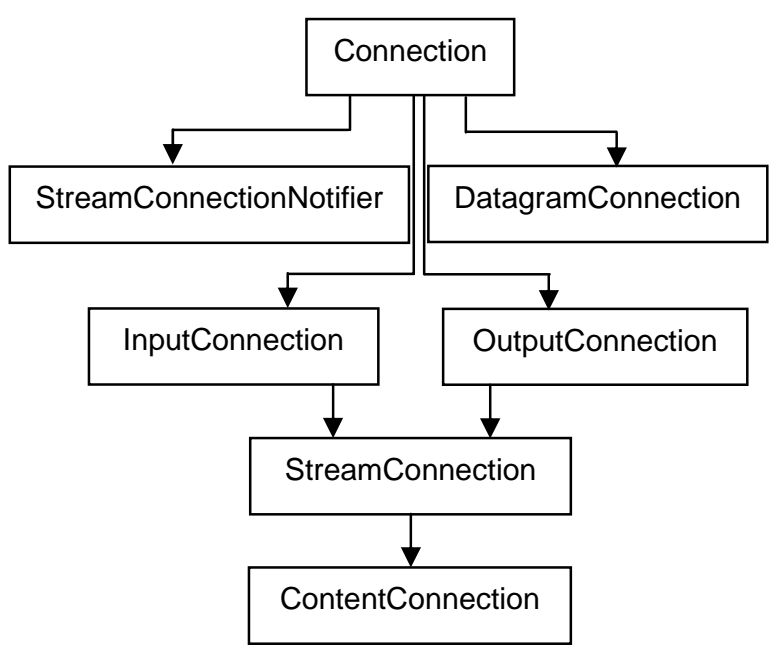

Figure 3: Arborescence of the classes javax.microedition.io.

The above diagram corresponds to the different classes javax.microedition.io. It is thanks to these implementations that we can make http requests.

The application on the client, called MIDlet, is carried out with the virtual machine J2ME (KVM) on the mobile terminal. It has the role to receive measurements of the devices of the patient at home, to treat these data or to store them if necessary.

It also allows to the doctor to send an alarm in case of a critical situation.

\subsection{Development on PC}

The second application (patient at home) is composed of different sub-programs. They have for function to store the entering data in a database, and allow to the doctor to establish the diagnosis of the patient either by execution of an algorithm incorporated into his cell phone, or by exploiting the received data.

\subsection{Simulation of proposed model}

The networks GSM/GPRS are useful to transmit ECG data of a patient. Currently, mobile phones of last generations are able to send and receive all sorts of messages (text, image, sound...). They offer in addition to the voice communication, a supply of services on a large scale, which allows considering a multitude of applications for these devices.

Our investigation consists to integrate an ECG signal on a mobile phone to ensure a medical remote monitoring service of patients at home. We describe in what follows the basic concept in electrophysiology of the heart in order to specify the components of the electrocardiogram (ECG).

\subsubsection{Electrocardiography}

Electrocardiography deals with the study of the electrical activity of the heart muscles.

The human body being electrically conductive, the electric potentials produced by the activity of the heart can be collected by electrodes placed on the thorax the model recorded of this electric activity of the heart, on a frontal level (derivations of the members) and on a horizontal level (derivations of precordiales) is an electrocardiogram.

For each heartbeat (Figure 4), the electrocardiogram record 4 successive waves ( $P, R, T$ and $U)$.

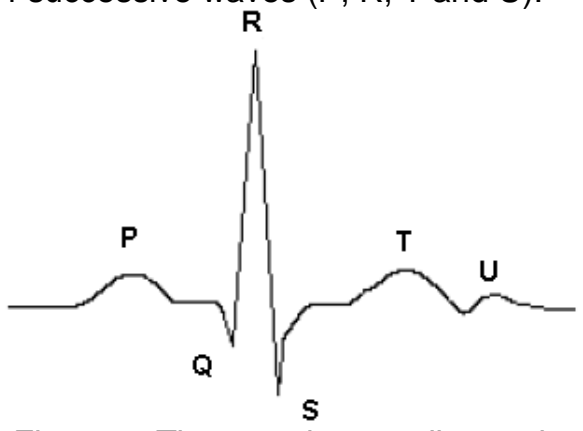

Figure 4: The complete cardiac cycle.

Each of these waves is characterized by its amplitude and its duration. The table 1 summarizes these values for a normal person [7].

Table 1: The parameters of an ECG signal.

\begin{tabular}{|c|c|c|c|}
\hline $\begin{array}{c}\text { Type } \\
\text { of } \\
\text { wave }\end{array}$ & Origin & $\begin{array}{l}\text { Amplitu- } \\
\text { de (mV) }\end{array}$ & $\begin{array}{l}\text { Durati- } \\
\text { on (Sec) }\end{array}$ \\
\hline $\begin{array}{c}\text { Wave } \\
\text { P }\end{array}$ & $\begin{array}{c}\text { Atrial } \\
\text { depolarization } \\
\text { or contraction }\end{array}$ & $<=0.2 \mathrm{mV}$ & $\begin{array}{c}\text { Interval : } \\
\text { P-R } \\
0.12 \\
\text { À } 0.22 \\
\end{array}$ \\
\hline $\begin{array}{c}\text { Wave } \\
\text { R }\end{array}$ & $\begin{array}{l}\text { Repolarisation } \\
\text { of the atria and } \\
\text { the } \\
\text { depolarization } \\
\text { of the } \\
\text { ventricles }\end{array}$ & 1.60 & $\begin{array}{c}0.07 \\
\text { À } 0.1\end{array}$ \\
\hline $\begin{array}{c}\text { Wave } \\
\mathrm{T}\end{array}$ & $\begin{array}{l}\text { Ventricular } \\
\text { repolarisation } \\
\text { (Relaxation } \\
\text { of myocardium) }\end{array}$ & $0.1-0.5$ & $\begin{array}{c}\text { Interval : } \\
\text { Q-T } \\
0.35 \\
\text { À } 0.44\end{array}$ \\
\hline $\begin{array}{l}\text { Inter- } \\
\text { val } \\
\mathrm{S}-\mathrm{T}\end{array}$ & $\begin{array}{l}\text { Ventricular } \\
\text { contraction }\end{array}$ & & $\begin{array}{c}\text { Interval: } \\
\text { S-T } \\
0.015 \\
\end{array}$ \\
\hline
\end{tabular}




\begin{tabular}{|c|c|c|c|}
\hline & & & À 0.5 \\
\hline & Slow & & \\
Wave & repolarisation & & \\
of the & Interventricular & $<0.1$ & Interval : \\
(Purkinje & T-U \\
& fibers) system. & & 0.2 \\
& & \\
\hline
\end{tabular}

This diagnostic tool allows detecting the rhythmic cardiac pathologies, muscular, extracardiaques metabolic problems, Medicinal, hémodynamiques and others.

\subsubsection{Application}

This section describes a medical application implemented in a mobile phone to treat and characterize the ECG signal.

Our application consists to develop a MIDlet to take remotely the evolution of the state of patients and to calculate parameters witch characterize the ECG signal.

The implementation of the proposed model for transmission simulating, storage and data processing of the electrocardiogram is realized with the J2ME environment. For reasons relating to the rapid evolution of technology, it is always preferable to avoid carrying out specific applications to a type of mobile equipment owner (Windows, Symbian, Palm / OS) [8]. J2ME allows the development of applications which can run on all compatible mobiles.

The following paragraphs present the principle of the implementation, the global structure of the implementation of simulation and finally the analysis result of the calculated parameters (Cardiac frequency averages, QRS duration ...).

\subsubsection{The principle of implementation}

Being given the complexity between the portable telephone's technology and number of parameters of the model which must be defined in priori, an adaptation of data to this context consists to use a set of files in the format text for the definition of the current exploited values for the simulation and the default values. A graphical interface on the phone allows the display of contained parameters in the files. The simulation, once launched, can then retrieve values from these files. At the end of the application, the generated results are stored in memory of the mobile terminal, and possibly displayed on the screen.

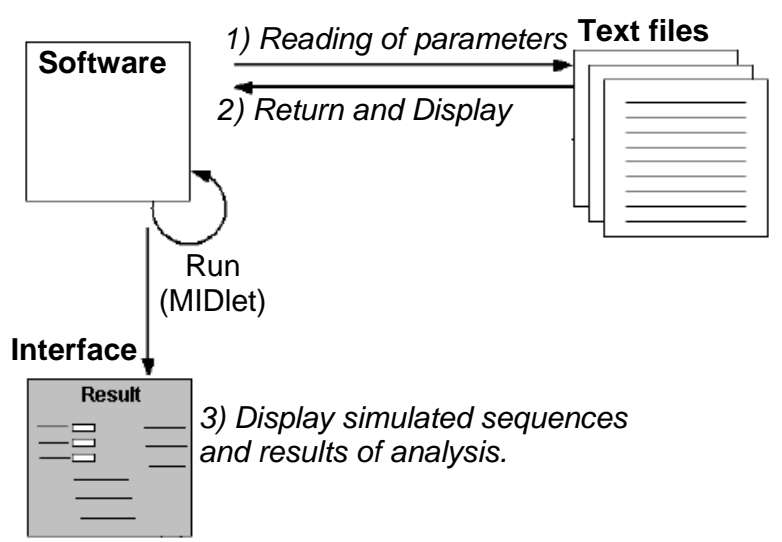

Figure 5: Principle of the process implementation of simulation.

The result interface is presented on Figure 8 and Figure 9. The parameters are calculated according to the simulation model sequences on which they involve.

\subsubsection{Global structure of the implementation of simulation}

The global structure of the simulation program is completely sequential. It calls, one after the other, the functions realizing the principle stages of the simulation and successively corresponding to the generation of the different parameters of ECG signal.

These parameters include the duration between two successive peaks R, complex duration QRS and cardiac frequency. Each called function takes as entering parameter the results of the call of the previous function and provides the results of its execution to the following function.

The stages of the MIDlet execution are detailed in the following section.

\subsubsection{Calculated parameters and analytical result}

As we have already seen before, J2ME wireless development was exploited to the implementation of the proposed simulation model of ECG signal on a mobile phone.

In this section, we present the various stages of execution of the algorithm. All this series of tests was made thanks to the phone emulator.

During the launching of the application, MIDlet allows to the doctor to activate the mode of the 
medical remote monitoring ECG (Remote Monitoring ECG):

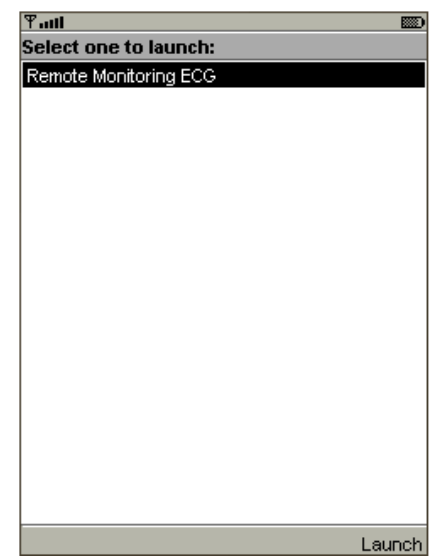

Figure 6: Launch of the algorithm.

At the beginning, the application will operate and communicate in autonomous mode with measurement devices. Then, the phone collects 5000 samples, generated by the electrodes placed on body of the patient at home (Figure 7, Figure 8) and stores these data.

These samples are points in $\mathrm{mV}$ which constitute the Electrocardiogram.

Project "ECG" loaded

Project settings saved

Building "ECG"

Build complete

Running with storage root DefaultcolorPhone

Running with locale: French_France.1252

(5000)

Figure 7: Acquisition of 5000 samples of a patient (ECG signal).

Figure 8 shows the organization in vectors of the ECG values transferred via GSM/GPRS to the internal memory of the mobile phone. They are obtained after amplification at domicile of patients with a gain factor of 1000 and a sampling frequency of $128 \mathrm{KHz}$.

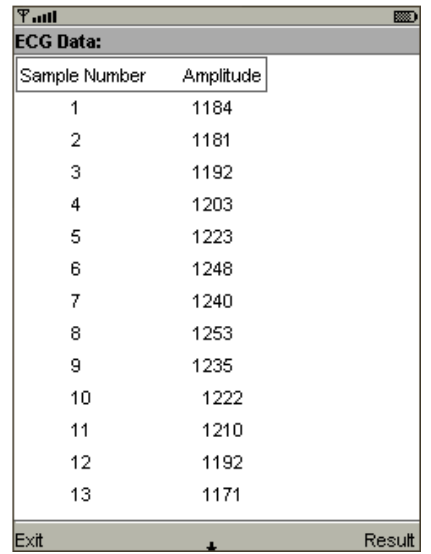

Figure 8: Data ECG transmitted to the mobile phones.

Such a medical application proposes a set of services to the health's professionals (list of the patients, the display of the medical profile of a patient...). These services make treatments with variable complexities (management of data via a data base, numeral calculation...) and exchange data with the user through a graphical interface on a mobile terminal. This environment type presents important and heterogeneous information, a great variability and numerous possibility of evolution. Indeed, the offered resources on the level of terminal can be extremely different according to the use of a personal assistant, a laptop or a workstation. Thus it is necessary to implement an adaptation strategy to conceive and develop the algorithm by respecting these prerequired constraints.

The graphical interface which is in this case the portable's screen, allows to collect, display, store and calculate the parameters of simulation (time between two successive peaks R, QRS duration,...), after an adaptation of medical data to the context. Our adaptation strategy consists to convert or replace the content of a text file to be sent to the doctor.

For example, we convert a character to a number because the values of the ECG are recorded as format ".Txt".

The principle of this process is the characterization of ECG signal. After receiving the medical data (Figure 8), the doctor is invited to consult the latest results of his patient (Figure 9) in order to take the adapted decision.

The diagnosis and treatment can be done using the implementation of calculation algorithm on the mobile. It allows calculating the most significant 
parameters necessary to the characterization of an ECG.

First of all the incorporated algorithm in the mobile phone must position all the peaks $\mathrm{R}$ (after an adaptation of recorded data in the textual files quoted before). It calculates the duration between each two successive peaks $R$ by multiplying the number of point located between these two peaks by the sampling frequency. To deduce the average cardiac frequency it is enough that it calculates the average of the durations of a fixed number of peaks $\mathrm{R}$ than it divides one fixed period (one minute in this case) by this average value (Figure 9 ).

The calculation of the QRS duration follows the same procedure. The last stage is the display of ECG signal on the mobile terminal's screen (Figure 10).

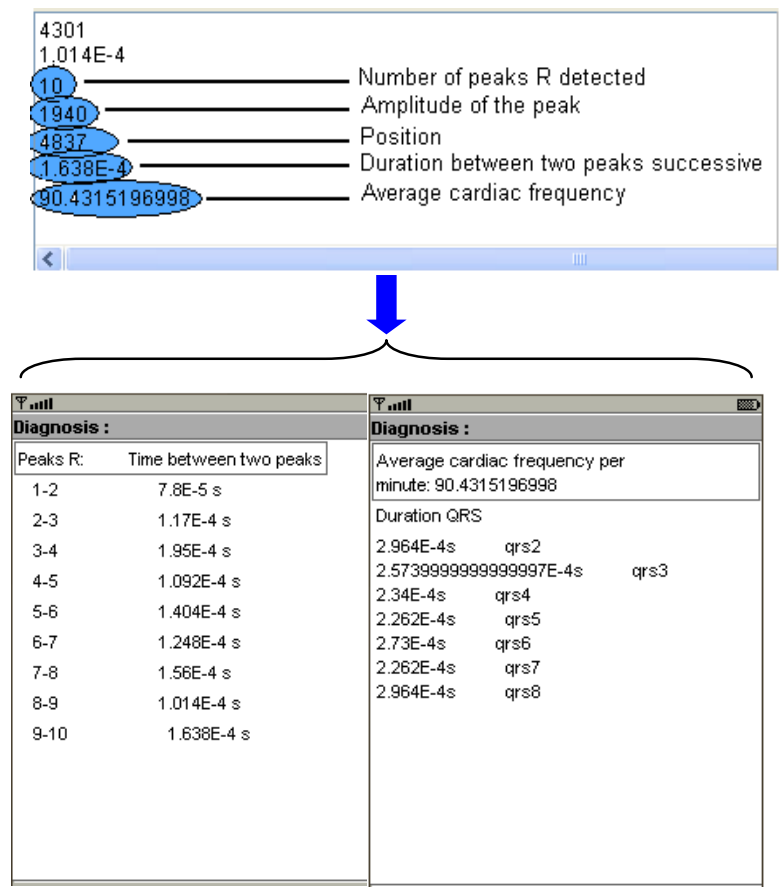

Figure 9: The calculated parameters.

In this scenario, it has involved several peaks $\mathrm{R}$ for the implementation of each model containing the definition of parameters. Each simulation model containing ECG data corresponding to a patient generates parameters which characterize the patient: time between two successive peaks, duration QRS and cardiac frequency. Thus, the doctor can observe the ECG signal in real time on his screen with 5000 samples: (Figure 10)

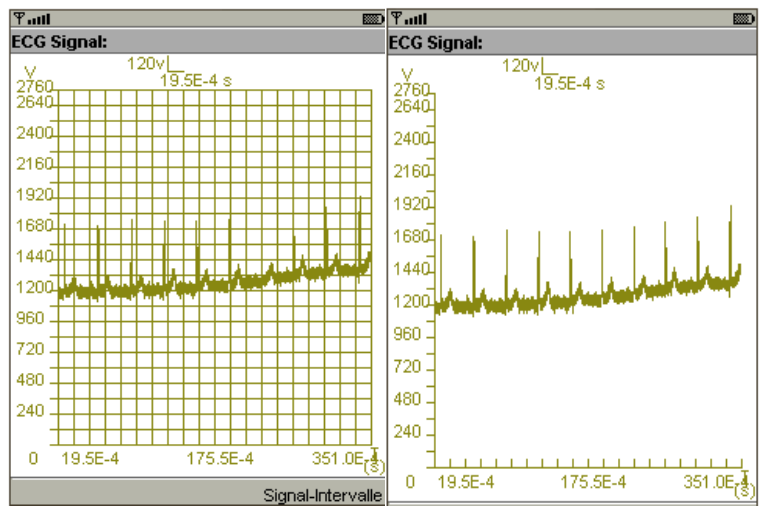

Figure 10: Show ECG waveform of the patient (with / without grid).

A more importantly option allowing the zoom of the part which presents an anomaly, is implemented in our application. It is enough to the doctor to introduce a begin point and an end point (time interval) in order to widen the part in question (Figure 11).

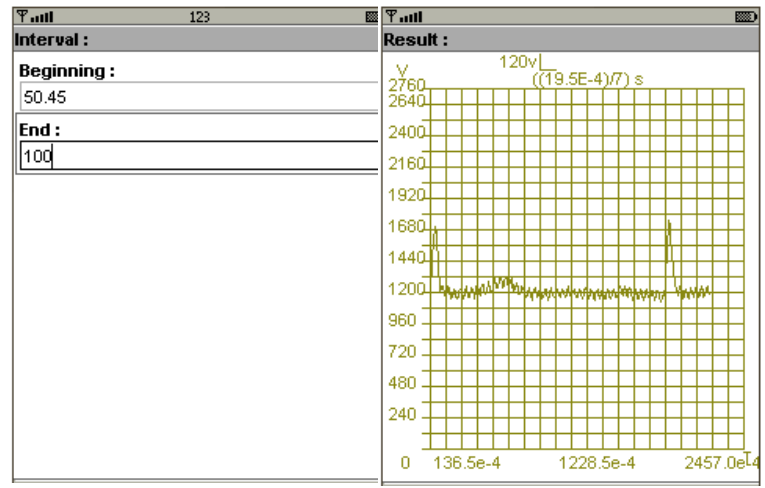

Figure 11: Zoom part of the curve.

At present we are interesting in the transmission of an ECG signal on several channels to make a multitude of analysis for the same cardiac patient and increase so the efficiency of the system, while having the possibility of revising all the results.

\subsubsection{Comparison with the simulation MATLAB}

The sequence data generated for the parameters of ECG in the context of medical remote monitoring (MIDlet) are validated by a comparison with simulated data under MATLAB environment (Figure 12). 


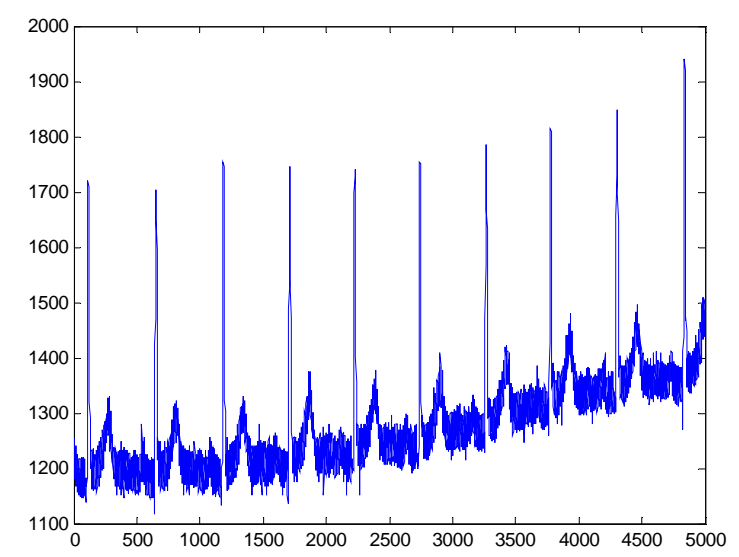

Figure 12: ECG signal.

The validation which is carried out about the implemented simulation process by the tool of J2ME development makes this algorithm to be exact in terms of calculation, powerful and effective. It was developed in the context of medical remote monitoring, from the recorded data sequences at home. The obtained results allow making advance the strengths points and the innovative qualities of this algorithm compared to other carried out works in similar domains or related, at the same time our algorithm presents better perspectives of improvement of its efficiency.

\subsection{Quality of service}

Within the framework of this work, the problem of the quality of service of the mobile networks for the second or third generation, lives on one hand in a environment with various requirements of good functioning and on the other hand in two ways of transport of the information: circuit or packet.

The doctor wants to know always about state of evolution of the patient. What requires to have at least an acceptable quality of service in the mobile communication to be able to sanction by a correct decision-making.

We can say that the zero risk does not exist. Prevention measures in the system of remote monitoring, chosen suitably according to needs, can reduce considerably this risk. For that purpose, this application requires a real-time transmission and is not tolerant in the errors. It requests for more or less raised debits.

\subsection{Instructions of the algorithm 4.5.1. Minimal configuration}

To be able to use the algorithm of analysis, one needs a mobile phone, which has the following characteristics:

- Support of profiles MIDP 2.0 or more and of CLDC 1.0 (or CLDC 1.1).

- Support of the api WMA 1.1 (JSR 120) and Web Services API (JSR 172).

- 60ko minimum of memory capacity available on the phone to store 5000 samples and the calculated parameters (after analysis).

\subsubsection{Diffusion of software}

To diffuse this software, there are several possibilities, but in each case, the two only files which must be provided to the user are: the file ".jad" and the file ".jar". These files must be transferred on the mobile phone, for that there are several solutions:

Download on the phone of an E-mail containing both files in attached peaces.

- Access to the files placed on a Web server.

- Transfer using Bluetooth or infrared signal.

In all the cases, the two files must be in the same one repertory or the same E-mail.

\subsubsection{Installation of the program}

Being given that the installation differs according to the mobile phones, it is not possible to give a precise procedure. But normally, the simple fact of opening the file ".jad" is enough to install the application.

\section{Evaluation}

The proposed simulation algorithm is articulated on two fundamental points: the first one relates to the simulation step in the respect of the complexity and objectives of the medical remote monitoring context at home, the second one is the more global vision in the resolution cycle of construction problem of the behavior profile of person to ensure a critical situation. The suggested remote monitoring consists to monitor and diagnose the state of a patient using the methodology developed in this project. Thus, the doctor treating a person with risk cardiac can at any time control the state of his patient by consulting in 
real-time the ECG on his mobile terminal and the classification of pathology by the developed algorithm.

\section{Conclusion}

This article refers to analysis biological signals of a patient, recorded at home and detected on the mobile phone of his doctor. This technique allows medical remote monitoring of cardiac or hypertensive patients.

Also, the identification, by the developed algorithm, of the medical profile of a patient at home and the detection of critical situations cannot cover all medical indicators corresponding to each patient.

Thus, the improvements of this algorithm must be adapted to diagnose new pathologies.

This solution, not costly and easily realizable, is adapted to the portable devices ensuring medical monitoring anytime and anywhere. It is in this vision that other services, associated to mobiles and intended for the telemedicine and the house automation are under development.

\section{Acknowledgement}

I deeply thank my supervisor and the person in charge of the laboratory STIC, Mohamed FEHAM, Professor at the university Abou Bekr Belkaid of Tlemcen, Algeria. The correctness of his advice, the motivation and the project financing. They were very precious and brought a successful conclusion to this work. A special thank to the researchers of the laboratory who gave their help to the realization of this project.

\section{References}

[1] Fouial, O. \& Demeure, .I. (2004) "Fourniture de services adaptables dans les environnements mobiles", in Proceedings of European Conference of Systems with adaptable and extensible component, Grenoble, France, 2004.

[2] Celler, B.G.; Hesketh, T.; Earnshaw, .W \& Ilsar, .E. (1994) "An instrumentation system for the remote monitoring of changes in functional health status of the elderly at home", in Proceedings of the 16th Annual IEEE Engineering in Medicine and Biology Society, Baltimore, USA, 1994, pp. 908-909.

[3] Chwif, L. \& Paul, R.J. (2000) "On simulation model complexity", in Proceedings of the 32nd conference on Winter simulation, Orlando, Florida, 2000, pp. 449-455.

[4] Delb, B. (2002) "J2ME, Application java pour terminaux mobiles", EYROLLES, Paris, France, 2002.

[5] Quintas. A. F. (2004) "Bluetooth J2ME Java 2 micro edition", Tutorial, Madrid: Ra-Ma, 2004.

[6] Knudsen, J. (2003) "Wireless Java Developing with J2ME", Second Edition, Apress, Berkeley, United States, 2003.

[7] R Legameta, R.; Addisson, P.S.; Grubb, N.; Robertson, CE.; Fox, K. \& Watson, J.N. (2003) "Real-Time Classification of ECGs on a PDA", IEEE Trans. On Information Technology in Biomedicine, vol. 30, 2003, pp. 565-568.

[8] Mahmoud. H, (2002) "Learning Wireless Java", O'Reilly, Sebastopol, USA, 2002. 


\section{Authors Biographies}

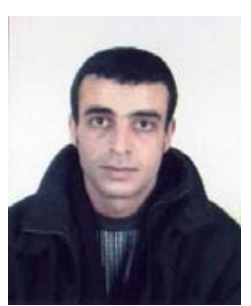

MERZOUGUI Rachid received the Master degree in Systems and Networks of Telecommunications from the University of Tlemcen (Algeria) in 2006. Since this year, he has been Assistant Professor of Mobile Networks and Services. He has served on the scientific committees of the Telecommunication Department of the University of Tlemcen. He is interested now in mobile services.

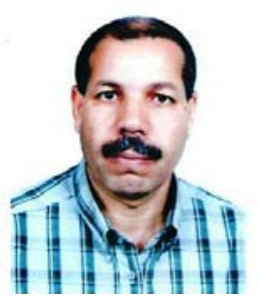

FEHAM Mohammed received the Dr. Eng. degree in optical and microwave communications from the University of Limoges (France) in 1987, and his PhD in Science from the University of Tlemcen (Algeria) in 1996. Since 1987, he has been Assistant Professor and Professor of microwave and communication Engineering. He has served on the Scientific Council and other committees of the Electronics and Telecommunication Departments of the University of Tlemcen. His research interest now is mobile networks and services. 